\title{
Design of multi-objective damping controller for gate-controlled series capacitor
}

\author{
AMIN SAFARI ${ }^{1, *}$ and NAVID REZAEI ${ }^{2}$ \\ ${ }^{1}$ Department of Electrical Engineering, Ahar branch, Islamic Azad University, \\ Ahar, Iran \\ ${ }^{2}$ Department of Electrical Engineering, Iran University of Science and Technology, \\ Tehran, Iran \\ e-mail: a-safari@iau-ahar.ac.ir
}

MS received 5 August 2012; revised 25 February 2013; accepted 6 September 2013

\begin{abstract}
This paper proposes an optimization procedure based on eigenvalues to carry out the stabilization function of the Gate-Controlled Series Capacitor (GCSC) in a power system. It is aimed to provide a reliable damping framework by means of a GCSC based multi-objective damping controller. The proposed method employs Particle Swarm Optimization (PSO) to search for optimal parameter settings of a widely used multi-objective lead-lag damping controller. The eigenvalue analysis is considered as the cornerstone of the performed studies in order to investigate the multi-objective methodology in which the unstable or lightly damped modes are scheduled to effectively shift to some prescribed stability zones in the s-plane. The effectiveness of the suggested approach in damping local and interarea oscillations modes in a multi-machine power system, over a wide range of loading conditions, is confirmed through eigenvalue analysis and time simulation.
\end{abstract}

Keywords. GCSC; PSO; Inter-area oscillation damping; multi-machine power system.

\section{Introduction}

The use of power system stabilizers (PSSs) for improving the dynamic stability of power networks has received increasing interest during the past three decades (Kundur 1994; Padiyar 2007). Recently, Flexible AC Transmission System (FACTS) technology has brought about a more universal stabilizing factor to the power systems. One of the most popular utilities of the FACTS devices is rapid controllability to provide a dynamic security limit in which, at least the system's inherent electromechanical oscillations, are mitigated guardedly. Hence, employing the FACTS controllers in such a stressed power system is crucial to the system's stability improvement (Hingorany \& Gyugyi 2000; Sadikovic 2006). Gate Controlled Series Capacitor

${ }^{*}$ For correspondence 
(GCSC), treated as a new contribution to the series of FACTS devices, is a potent power modulator producing controllable series compensation (Karady et al 1993). A single module of a GCSC comprises of a capacitor connecting in shunt, with a pair of anti-parallel gate-commutated switches. Wide range of controllability is the direct result of the gate-commutated based scheme. Furthermore, the GCSC with a structural-based restraining of the resonant frequencies, unlike that which occurs in the case of Thyristor Controlled Series Capacitor (TCSC), provides a continuous stabilizing signal standing to offer a more beneficial power system operation. For the sake of comparison to especially the TCSC and Static Synchronous Series Compensator (SSSC), simplicity (De Souza et al 2007) and high efficiency are the main features that enable the GCSC to be considered as a powerful option in enhancing the dynamic stability (Ray et al 2008). To augment the modulated damping torque of the GCSC against the system uncertainties and nonlinear interactions and to attain a more techno-economical operation of the GCSC modules, a supplementary damping controller is proposed and designed in a way in which the GCSC stabilizing signal is reinforced. As reported in literature, to improve a better damping of power system, some commonly used modern control methodologies such as neural networks (Ray et al 2008) and fuzzy set (Alizadeh Pahlavani \& Mohammadpour 2011) are developed to design a GCSC based single objective damping controller. The present study uses Particle Swarm Optimization (PSO) algorithm to search for the optimistic set of damping controller parameters using of multi-objective fitness function. Because of the impossible nature of the PSO to the particularly nonlinear, differential and large scale problems (Wanatabe et al 2007; Del Valle et al 2008; Shayeghi et al 2010), and further referring to the multi-modal fulfillment of the controller parameters, it seemed to be an appropriate candidate to solve the problem of controller design. The optimal design of the controller parameters is converted into a minimization problem with eigenvalue based multi-objective fitness function and solved by PSO algorithm. The purpose is to shift the un-damped or lightly damped eigenvalues to some pre-specified stable regions in the s-plane. Besides, to guarantee the robustness and competency of the multi-objective damping controller, a wide range of operating points is assumed to be applied to the test power system.

\section{Gate controlled series capacitor}

According to the substantial effects of the GCSC in increasing the power system available transfer capability and thereby, providing more developed system stability margins, it is necessary to exploit a detailed and as well as a compatible model to the nonlinear ingredients of a practical large scale power system. In this quest, the current injection model of the GCSC is drawn out through the variable impedance model. Truism, as represented in figure 1 for the GCSC installed between the buses $i$ and $j$, the equivalent impedance is a variable capacitance changeable by the switch blocking angle variations. It is notable that the activate series compensation is produced when the blocking angle varies in the range of 0 to 90 degrees with respect to the line current maximum point (Hingorany \& Gyugyi 2000).

Eq. (1) explains the relationship between the GCSC capacitive reactance and the blocking angle $\gamma$ (Alizadeh Pahlavani \& Mohammadpour 2011; Hingorany \& Gyugyi 2000; De Souza et al 2007).

$$
X_{G C S C}(\gamma)=X_{C}\left(1-\frac{2 \gamma}{\pi}-\frac{\sin (2 \gamma)}{\pi}\right) .
$$

In figure 2, the equivalent impedance model of the GCSC is shown. 


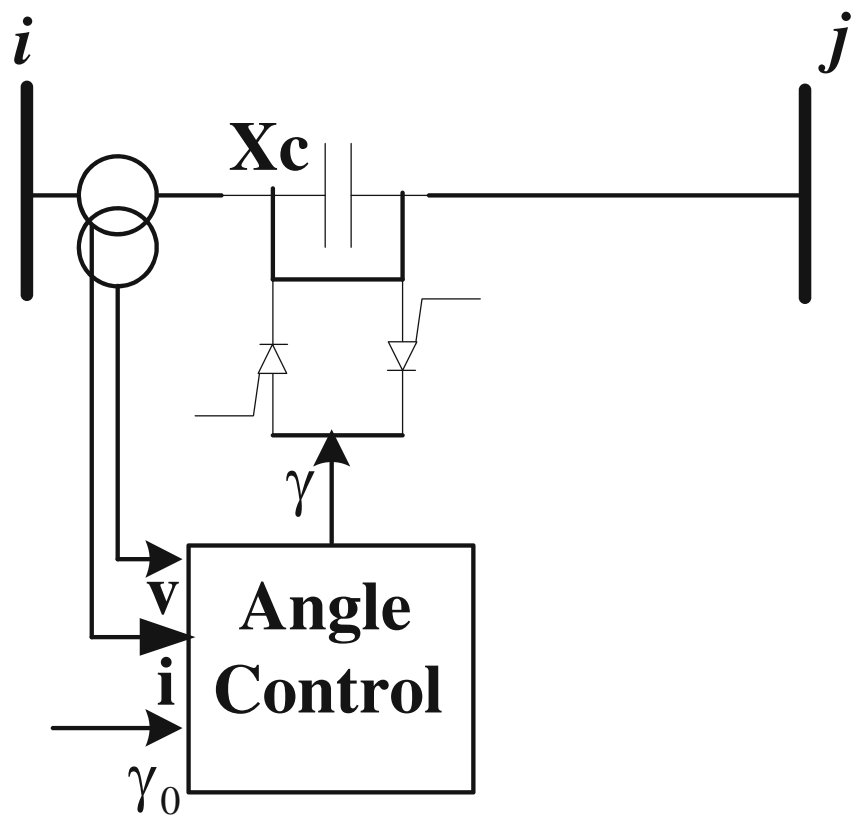

Figure 1. The GCSC installed between buses $i$ and $j$.

To attain the proposed current injection model, first the series current, which flows through the compensated transmission line, is considered to be calculated according to figure 2 as following:

$$
\bar{I}_{s e}=\frac{\bar{V}_{i}-\bar{V}_{j}}{r_{l}+j\left(x_{l}-x_{G C S C}(\gamma)\right)},
$$

where, the voltage phasors of the buses $i$ and $j$, the resistance and reactance of the transmission line and the variable GCSC reactance are represented by $\bar{V}_{i}, \bar{V}_{j}, r_{l}, x_{l}$ and $x_{G C S C}(\gamma)$, respectively.

The next stage is to replace the series variable voltage source $\left(\bar{V}_{S}\right)$ by an equivalent shunt current source as shown in figure 3. Finally, by calculating the injected current of the buses $i$ and $j$, the current injection model is extracted. Figure $3 b$ represents the final version of the GCSC current injection model. The calculations are as follows:

$$
\bar{V}_{s}=-j x_{G C S C} \bar{I}_{s e},
$$

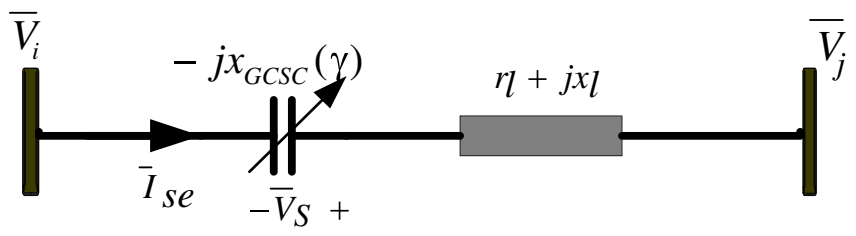

Figure 2. The equivalent circuit of the GCSC installed between buses $i$ and $j$. 


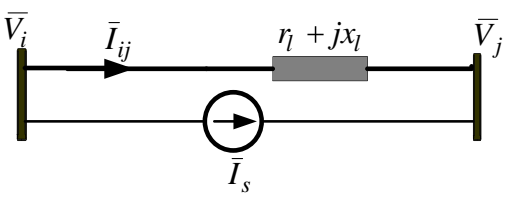

a

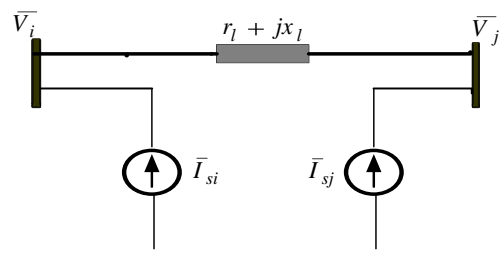

b

Figure 3. The GCSC current injection model. (a) Substitution of the shunt current source, (b) extraction of the current injection sources.

$$
\begin{gathered}
\bar{I}_{s}=\frac{\bar{V}_{s}}{r_{l}+j x_{l}}=\frac{-j x_{G C S C} \bar{I}_{s e}}{r_{l}+j x_{l}}, \\
\bar{I}_{s i}=\frac{-j x_{G C S C}}{r_{l}+j x_{l}} \cdot \frac{\bar{V}_{i}-\bar{V}_{j}}{r_{l}+j\left(x_{l}-x_{G C S C}\right)}, \\
\bar{I}_{s i}=-\bar{I}_{s j} .
\end{gathered}
$$

Notably, the derived current injected based model preserves the system nominal impedance matrix and consequently facilitates the process of nonlinear dynamic simulations (Sadikovic 2006; Shayeghi et al 2010).

\section{Design of GCSC multi-objective damping controller}

\subsection{The structure of the supplementary damping controller}

For the sake of upgrading the efficiency of the GCSC in producing an intensified damping torque, there is a need to employ an assistant damping controller with the aim of achieving an economical operation of the GCSC. It is notable that the classic lead-lag controllers are still preferred by the electrical utilities for their simplicity (Panda et al 2008). A signal washout block, a gain block and two-stage phase compensator block are the foundational construction of the commonly used supplementary controller. The main scope of the controller design is to provide a robust phase lead compensator to make up for the phase lag between input and output signals (Panda 2009). This implies optimal determination of $K, T_{1}, T_{2}, T_{3}$ and $T_{4}$ parameters. The block-diagram of the utilized GCSC-based damping controller is depicted in figure 4. $T_{W}$ is the washout time constant which typically takes a value in the range of 1 to 20 seconds (Shayeghi et al 2010) and here it is set within 10 seconds. $T_{\mathrm{GCSC}}$ denotes the natural time delay of the

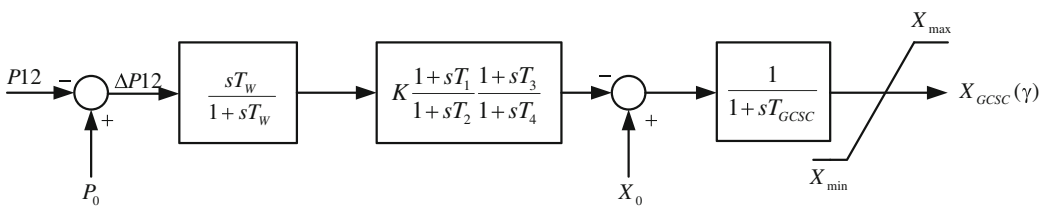

Figure 4. The structure of the proposed damping controller. 
blocking angle inside the controller impacted in the GCSC module and is selected within $20 \mathrm{~ms}$. $X_{0}, X_{\min }$ and $X_{\max }$ represent the GCSC reactance at initial set-point corresponding to the angle $\gamma_{0}$, lower limit of the GCSC reactance matched to the angle $\gamma=90^{\circ}$ and upper limit of the GCSC reactance rises from the angle $\gamma=0^{\circ}$, respectively.

The time-line active power $\left(\mathrm{P}_{12}\right)$, transferred between the two areas is selected here to be used as the proposed controller input signal. For this reason, this local signal contains invaluable information about the inter-area modes and is more adaptable to the application of the FACTS series controllers (Shayeghi et al 2010).

\subsection{Multi-machine test power system}

To investigate the damping performance of the GCSC, particularly regarding the mitigation of the inter-area oscillations, a four machine two area test power system, as shown in figure 5, is assumed to be simulated. The system encompasses four machines called G1, G2, G3 and G4 with similar ratings. The two distinct areas are apiece included two machines and connected through some parallel tie-lines within $413 \mathrm{MW}$ power transfer capacity from area 1 to area 2 . For simplicity and more adjustability, the operated loads are modelled as constant impedances and the damping impacts of the local PSSs are neglected. A forth-order dynamic model for each of the machines, each of them equipped with a static automatic voltage (AVR) controller, is utilized to be simulated and the GCSC is connected using the proposed current injection model between the buses 7 and 8. The system relevant data is given by Kundur (1994). The simulated nonlinear dynamics of the $i$ th machine are (Kundur 1994; Shayeghi et al 2010):

$$
\begin{gathered}
\dot{\delta}_{i}=\omega_{0}\left(\omega_{i}-1\right), \\
\dot{\omega}_{i}=\frac{P_{m i}-P_{e i}-D_{i}\left(\omega_{i}-1\right)}{M_{i}}, \\
\dot{E}_{q i}^{\prime}=\frac{E_{f d i}-\left(x_{d i}-x_{d i}^{\prime}\right) i_{d i}-E_{q i}^{\prime}}{T_{d o i}^{\prime}}, \\
\dot{E}_{f d i}=\frac{K_{A i}\left(V_{r e f i}-V_{t i}\right)-E_{f d i}}{T_{A i}}, \\
T_{e i}=E_{q i}^{\prime} i_{q i}-\left(x_{d i}-x_{d i}^{\prime}\right) i_{d i} i_{q i}
\end{gathered}
$$

where, $\delta, \omega, P_{m}, P_{e}, E_{q}^{\prime}, E_{f d}, T_{e}, T_{d o}^{\prime}, K_{A}, T_{A}, V_{r e f}$ and $V_{t}$ denote rotor angle, rotor speed, mechanical input power, electrical output power, internal voltage behind $x_{d}^{\prime}$, equivalent

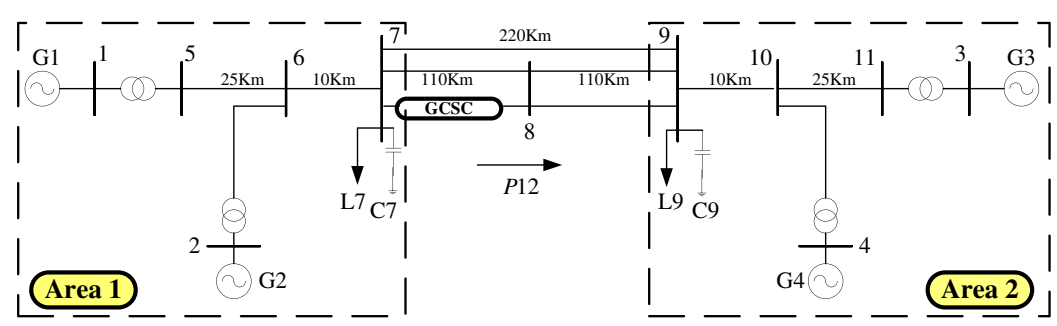

Figure 5. The two area four machine test power system equipped with the GCSC. 
excitation voltage, electric torque, time constant of excitation circuit, AVR gain, AVR time constant, reference voltage and terminal voltage, respectively. Besides, it is worth mentioning that all of the simulation processes from mathematical modelling from current injection model, to the implementation of the proposed PSO-based GCSC damping of the controllers in the test power system, are performed in the MATLAB/SIMULINK environment.

\subsection{Multi-objective formulation}

As mentioned earlier, the problem of the proposed damping controller design is multi-modal which means that it has more than one local optimum solution. Therefore, it is appropriate to employ an adequate optimization algorithm, to find the global optimal solution to the design problem. In this study, PSO algorithm was utilized to solve the optimization. Thus, the problem of determining the set of damping controller parameters is transmitted to minimization of some eigenvalue-based fitness functions, in which the purpose is to suitably shift the unstable or lightly damped inter-area eigenvalue to some robust stable areas in the s-plane. It may be mentioned here that the eigenvalue analysis is currently an efficient indicator of the power system stability issues. The fitness functions are specified as the following $F_{1}, F_{2}$ and $F_{3}$ functions:

$$
\begin{gathered}
F_{1}=\sum_{i=1}^{N P} \sum_{\sigma_{k} \geq \sigma_{0}}\left(\sigma_{k, i}-\sigma_{0}\right)^{2} ; \sigma_{k, i}=\operatorname{Real}\left(\lambda_{k, i}\right), \\
F_{2}=\sum_{i=1}^{N P} \sum_{\xi_{k} \leq \xi_{0}}\left(\xi_{k, i}-\xi_{0}\right)^{2} ; \xi_{k, i}=-\operatorname{Real}\left(\lambda_{k, i}\right) /\left|\lambda_{k, i}\right|, \\
F_{3}=\sum_{i=1}^{N P}\left[\sum_{\sigma_{k} \geq \sigma_{0}}\left(\sigma_{k, i}-\sigma_{0}\right)^{2}+\rho \cdot \sum_{\xi_{k} \leq \xi_{0}}\left(\xi_{k, i}-\xi_{0}\right)^{2}\right] ; \rho=10,
\end{gathered}
$$

where, $\lambda_{k, i}, \sigma_{k . i}, \xi_{k, i}$ are $k$ th eigenvalue, real part of the $k$ th eigenvalue and the damping ratio of the $k$ th eigenvalue, all in the $i$ th operating point, respectively. The $\sigma_{0}$ and $\xi_{0}$ are pre-specified real part and damping ratio which are assumed to lead the system unstable and lightly damped inter-area eigenvalues to the considered stability areas depicted in figure 6 . The values of $\sigma_{0}$ and $\xi_{0}$ are -2 and 0.45 in this study. $N P$ represents the total number of operating points assumed to be carrying out the system performance. Meanwhile, it is scheduled to match the $F_{1}, F_{2}$ and $F_{3}$ functions to the areas demonstrated in figures $6 \mathrm{a}-\mathrm{c}$, respectively. Moreover, to achieve global optimistic solution, it is necessary that the PSO is performed under a wide range of operating

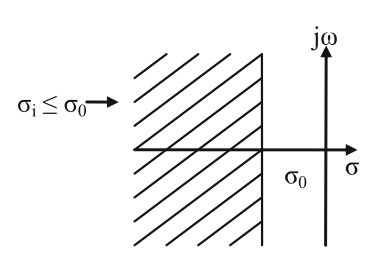

a

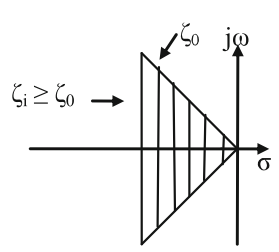

b

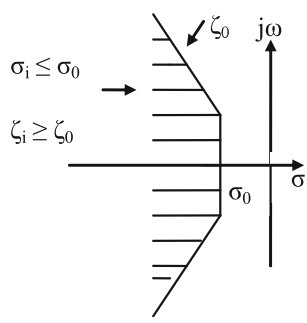

C

Figure 6. Regions of eigenvalue locations for different objective functions. 
Table 1. The considered power system operating points.

\begin{tabular}{lcccccccc}
\hline Operating point & $\mathrm{P}_{1}$ & $\mathrm{Q}_{1}$ & $\mathrm{P}_{2}$ & $\mathrm{Q}_{2}$ & $\mathrm{P}_{3}$ & $\mathrm{Q}_{3}$ & $\mathrm{P}_{4}$ & $\mathrm{Q}_{4}$ \\
\hline Case 1 & 0.7621 & 0.0835 & 0.6396 & 0.0571 & 0.8006 & 0.1131 & 0.7927 & 0.1362 \\
Case 2 & 0.9061 & 0.1639 & 0.7667 & 0.1934 & 0.7333 & 0.0732 & 0.7000 & 0.0764 \\
Case 3 & 0.5050 & 0.0519 & 0.9067 & 0.1155 & 0.8889 & 0.1163 & 0.8778 & 0.1065 \\
\hline
\end{tabular}

conditions. Table 1 gives the assumed operating points obtained using the multi-machine load flow analysis.

It is considered that the aforementioned fitness functions be optimized subject to the following bounded constraints:

$$
\begin{gathered}
0<K<200, \\
0.01<T_{x}<2, x=1,2,3,4,
\end{gathered}
$$

where, $\mathrm{K}$ and $\mathrm{T}_{x}(x=1,2,3,4)$ are the gain and lead-lag block parameters, respectively, as they have been represented in the damping controller structure in figure 4 .

\subsection{Review of the PSO algorithm}

Eberhart \& Kennedy (1995) constructed an evolutionary, stochastical optimization methodology, in which the main agenda was an intelligent mimic from the gregarious cooperation between the particles of a swarm of creatures, in order to attain the group profit. After that, due to the easier implementation, fewer adjustable parameters, efficiency in remaindering the last best performance, independent of the initial state conditions and sustainability in swarm diversification, it has been converted to a highly special solver by which a vast range of optimization chores

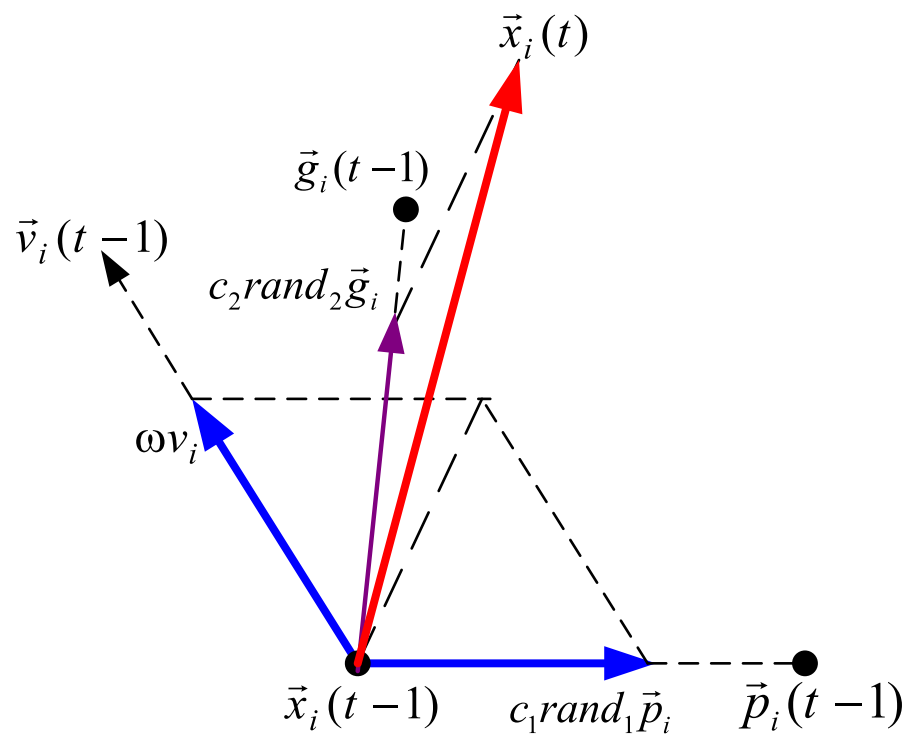

Figure 7. The search process of a 2-dimensional particle. 


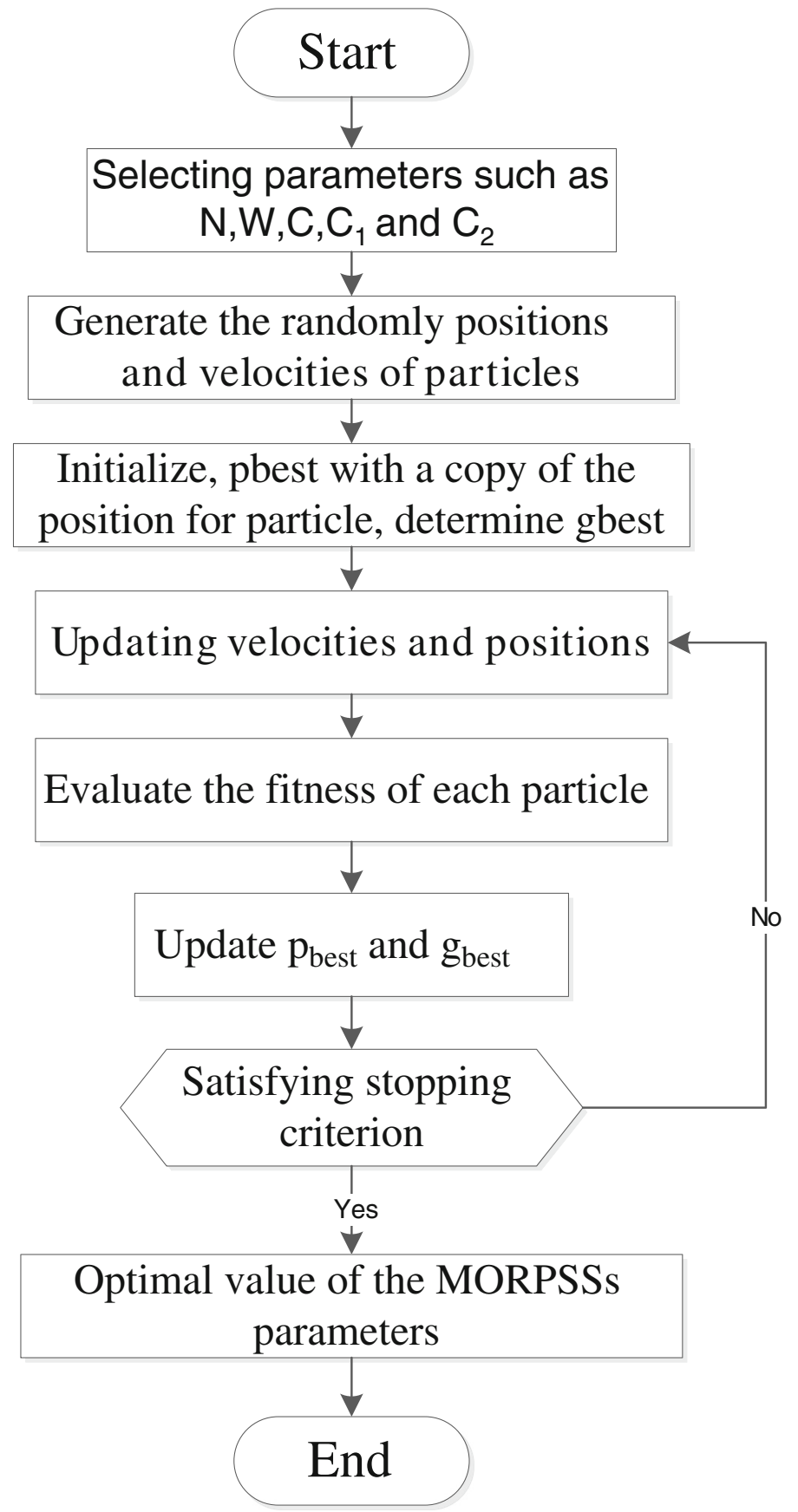

Figure 8. Flowchart of the optimization technique using PSO. 
could be suitably relieved (Shi 2008; Ho et al 2005). The particles, which are drifted in some multi-dimensional search hyperspaces, are capable of memorizing both the best individual (local optimum) and social (global optimum) positions experienced throughout each attempt, to seek for converge. This helps the swarm to recuperate from its exploration to pour towards the global optimum. In a D-dimensional search space, the position and velocity vectors of the swarm $i$ th particle are shown by $X_{i}=\left[x_{i 1}, x_{i 2}, \ldots, x_{i D}\right]$ and $V_{i}=\left[v_{i 1}, v_{i 2}, \ldots, v_{i D}\right]$. The fittest solutions for each particle and the overall swarm, achieved so far, are stored in pbest $=P_{i}=\left[p_{i 1}, p_{i 2}, \ldots, p_{i D}\right]$ and gbest $=P_{g d}$, respectively. $P_{g d}$ is the only best value among the swarm population. The agenda makes the swarm move toward the pbest and eventually the gbest. Thus the particles are accelerated besides updating the previous directions with respect to the pbest and gbest in Eqs. (16) and (17). Eberhart \& Kennedy (1995) describe this optimization procedure. Furthermore, in figure 7 , the intelligent exploration process of a 2-dimension particle in a sample hyperspace is illustrated.

$$
\begin{gathered}
v_{i d}(t+1)=\omega v_{i d}(t)+c_{1} \operatorname{rand}_{1}\left(\operatorname{pbest}(t)-x_{i d}(t)\right)+c_{2} \operatorname{rand}_{2}\left(\operatorname{gbest}(t)-x_{i d}(t)\right), \\
\vec{x}(t+1)=\vec{x}(t)+\vec{v}(t+1),
\end{gathered}
$$

where, $x_{i d}$, $v_{i d}$, pbest and gbest identify the position, velocity, local optimum of each particle and global optimum of the group, respectively (Eberhart \& Kennedy 1995). A random function rand is to generate the candidate solutions in each iteration. $c_{1}$ and $c_{2}$ are cognitive and social acceleration coefficients, respectively by which the adaptability with the global best particle is clearly indicated. To control the convergence ratio, especially in the last iterations, an inertia
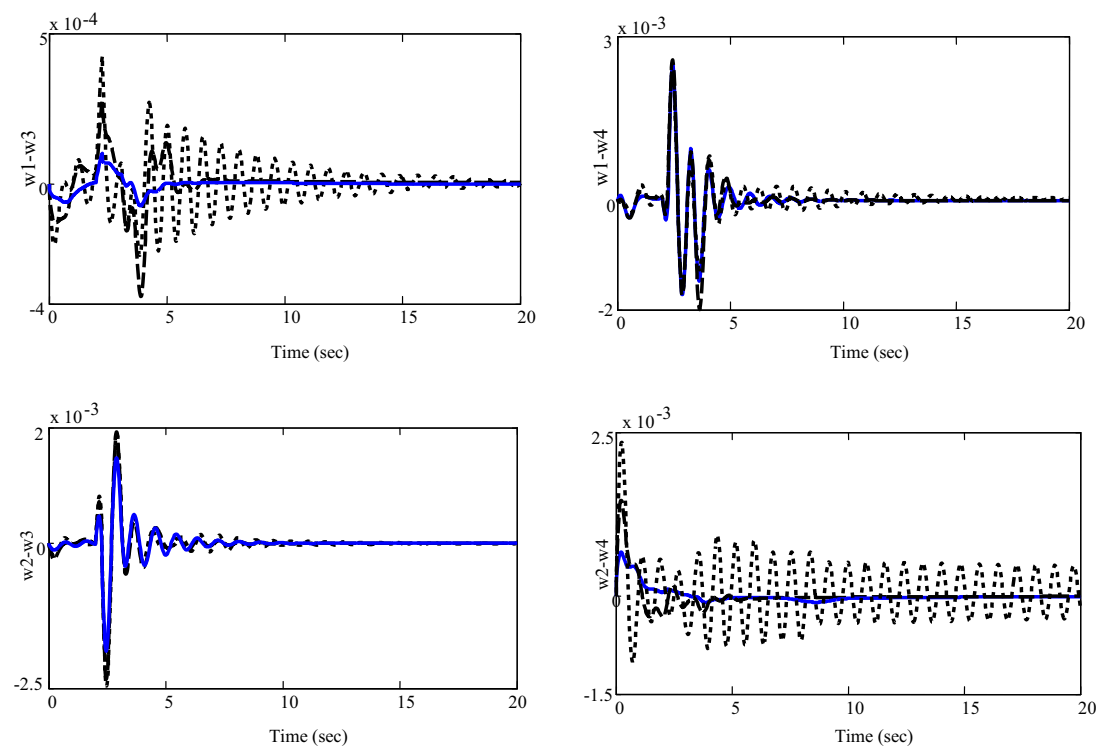

Figure 9. The power system inter-area rotor speed responses to the disturbance in Case 1: Solid $\left(F_{3}\right)$, Dashed $\left(F_{2}\right)$ and Dotted $\left(F_{1}\right)$. 
Table 2. PSO main parameters identification.

\begin{tabular}{lc}
\hline Main parameter & Value \\
\hline Number of the swarm particles & 50 \\
Number of iterations & 100 \\
Particle dimensions & 5 \\
$c_{1}$ (cognitive coefficient) & 2 \\
$c_{2}$ (social coefficient) & 2 \\
\hline
\end{tabular}

weight is set as $\omega$ in Eq. (16). The value of $\omega$ is decreased linearly from 0.9 to 0.4 during the assumed iterations (Nafar et al 2011):

$$
\omega=\omega_{\max }-\frac{\omega_{\max }-\omega_{\min }}{\text { iter }_{\max }} \text {.iter } .
$$

Figures 8 show the flowchart of the PSO algorithm. In this study, the parameters of the PSO are selected as given in table 2 .

\section{Simulation results}

In this study, the process of the GCSC multi-objective damping controller design is formulated to an optimization problem, which is proposed to be solved by the PSO algorithm. In order to determine the optimistic corresponding values of each particle and on the basis of the eigenvalue analysis, the value of each $F_{1}, F_{2}$ and $F_{3}$ fitness functions for each particle under various operating conditions is calculated. The parameters of controller, i.e., $K, T_{1}, T_{2}, T_{3}$ and $T_{4}$ which are optimized via the PSO algorithm, in order to provide an efficient power system stability operation are listed in table 3 , which indicate the optimized set of the damping controller parameters which are carried out for the $F_{1}, F_{2}$ and $F_{3}$ functions and concern the given operating points in table 1.

Also, the shifted inter-area eigenvalues corresponding to the $F_{1}, F_{2}$ and $F_{3}$ functions with their frequency and damping ratios are shown in table 4 . The criteria for the specified stability regions as illustrated in figure 6 have all been satisfied in the optimization procedure.

Moreover, to better assess the robustness of the multi-objective damping controller, it is assumed that a severe disturbance is imposed to the test power system. In this disturbance, an attempt was made to investigate the system performance under a more disturbance condition. Thus, a three-phase fault is assumed to have occurred at $t=2 \mathrm{~s}$ and in the middle of the transmission line between the buses 7 and 9 . The fault was cleared after $250 \mathrm{~ms}$ and by permanently tripping of the faulty line. To validate the simulated results of the eigenvalue analysis, the time simulation was taken into account. Figures 8 and 9 represent the time-line power transfer variations (P12) in response to the assumed disturbance and in the considered operating conditions.

Table 3. Optimized set of damping controller parameters for different fitness functions.

\begin{tabular}{lccccr}
\hline Fitness functions & $K$ & $T_{1}$ & $T_{2}$ & $T_{3}$ & $T_{4}$ \\
\hline$F_{1}$ & 87.12 & 0.0751 & 0.6610 & 0.9855 & 0.9003 \\
$F_{2}$ & 40.01 & 1.2556 & 0.0182 & 0.2116 & 1.0280 \\
$F_{3}$ & 194.63 & 0.0859 & 0.0442 & 1.6237 & 1.9025 \\
\hline
\end{tabular}




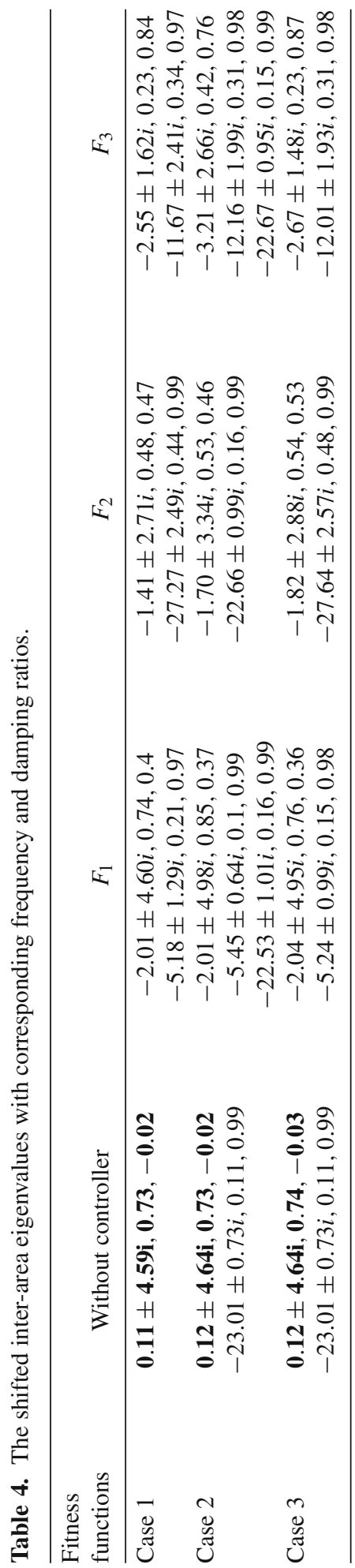



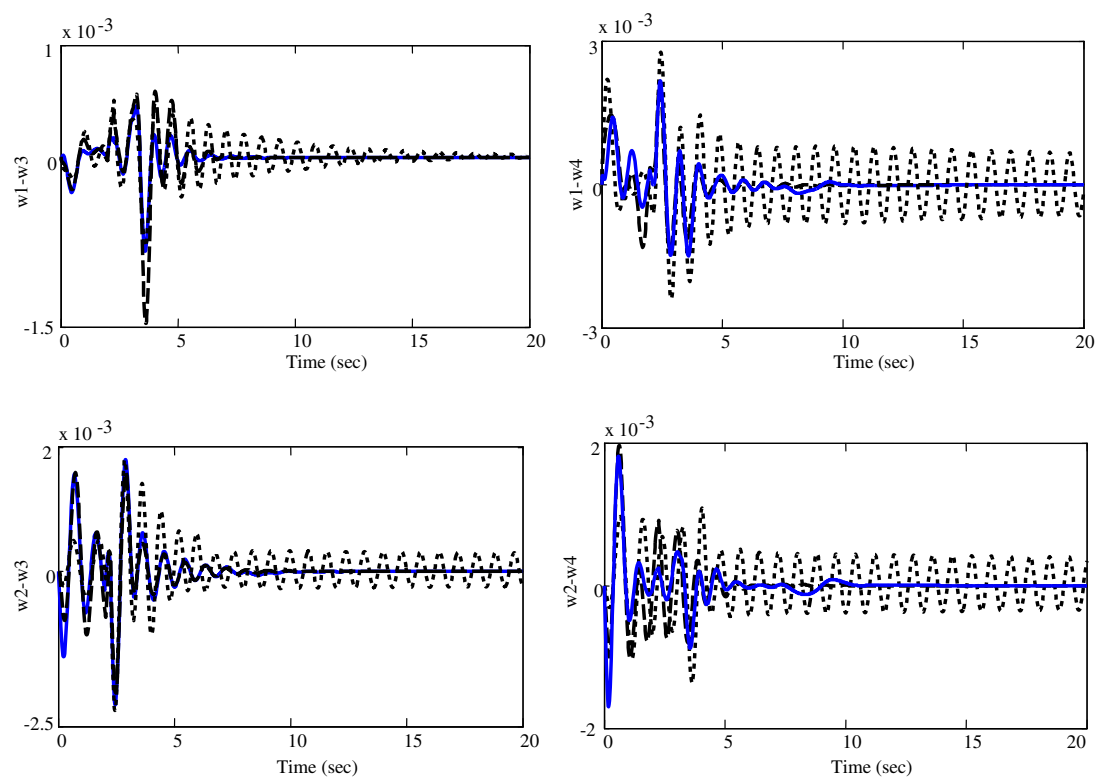

Figure 10. The power system inter-area rotor speed responses to the disturbance in Case 2: Solid $\left(F_{3}\right)$, Dashed $\left(F_{2}\right)$ and Dotted $\left(F_{1}\right)$.
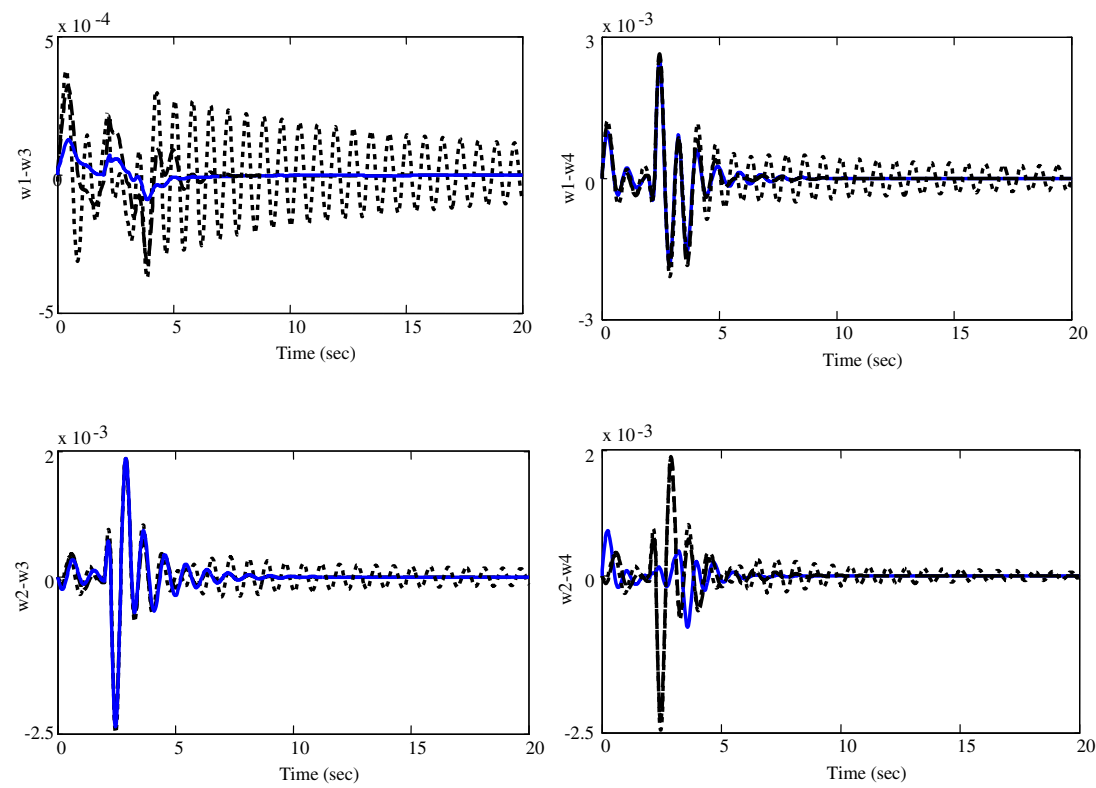

Figure 11. The power system inter-area rotor speed responses to the disturbance in Case 3: Solid $\left(F_{3}\right)$, Dashed $\left(F_{2}\right)$ and Dotted $\left(F_{1}\right)$. 
Furthermore, the relative inter-area machine speed deviations are depicted in figures 10 and 11. The optimized GCSC based damping framework is invaluable in providing more strengthened power system stability criterion, especially to the inter-area modes.

\section{Conclusions}

In this study, the gate-controlled series capacitor, as a potent FACTS device is considered in order to thoroughly investigate its capability in modification of the line reactance and providing enough damping to system oscillation modes. The results are given as follows:

- The mathematical analysis and current injection modelling of the gate-controlled series capacitor is presented.

- A current injection model of the GCSC for low frequency oscillations is proposed for the first time.

- The proposed model of the GCSC is explained mathematically. It can be implemented using an appropriate simulation tool and can be extended further for different applications in two area four machine power system.

- The problem of GCSC multi-objective damping controller is formulated as an optimization problem which is solved using PSO technique.

- The simulation results have shown that the designed multi-objective damping controller can effectively damp the power oscillations under sever disturbances.

\section{References}

Alizadeh Pahlavani M R and Mohammadpour H A 2011 Damping of sub-synchronous resonance and low frequency power oscillations in a series-compensated transmission line using gate-commutated series capacitor. Elect. Power Syst. Res. 81(2): 308-317

De Souza L F W, Wanatabe E and De Jesus D 2007 SSR and power oscillation damping using gatecontrolled series capacitors. IEEE Trans. Power Deliv. 22(3): 1806-1812

Del Valle Y, Venayagamoorthy G K, Mohagheghi S, Carlos J and Harlry R G 2008 Particle swarm optimization: Basic concepts, variants and application in power systems. IEEE Trans. Evol. Comp. 12(2): $171-195$

Eberhart R and Kennedy J 1995 A new optimizer using particle swarm theory. Micro machine and Human Sci. 39-43

Hingorany N and Gyugyi L 2000 Understanding FACTS: Concepts and technology of Flexible AC Transmission Systems, IEEE Press

Ho S L, Yang S, Ni G, Lo E W C and Wong H C 2005 A particle swarm optimization based method for multiobjective design optimizations. IEEE Trans. Magnetics. 41(5): 1756-1759

Karady G G, Ortmeyer T H, Pilvelait B R and Maratukulam D 1993 Continuously regulated series capacitor. IEEE Trans. Power Deliv. 8(3): 1384-1354

Kundur P 1994 Power system stability and control New York: McGrew Hill

Nafar M, Gharehpetian G B and Niknam T 2011 A novel parameter estimation method for metal oxide surge arrester models. Sadhana 36(6): 941-961

Padiyar K R 2007 FACTS controllers in power transmission and distribution, New age publishers, India

Panda S 2009 Multi-objective evolutionary algorithm for SSSC-based controller design. Elect. Power Syst. Res. 79: 937-944

Panda S, Padhy N P and Patel R N 2008 Power system stability improvement by PSO optimized SSSCbased damping controller. Elect. Power Comp. Syst. 36(5): 468-490 
Ray S, Venayagamoorthy G K and Watanabe E H 2008 A computational approach to optimal damping controller design for a GCSC. IEEE Trans. Power Deliv. 23(3): 1673-1681

Sadikovic R 2006 Use of FACTS devices for power flow control and damping of oscillations in power systems, $\mathrm{Ph}$. D. Thesis, Swiss federal institute of technology

Shi X Y 2008 Introduction to mathematical optimization from linear programming to meta-heuristics, Cambridge international science publishing

Shayeghi H, Shayanfar H A, Jalilizadeh S and Safari A 2010 TCSC robust damping controller design based on particle swarm optimization for a multi-machine power system. Energy Convers. manage. 51(10): $1873-1882$

Wanatabe E H, Aredes M, Barbosa P G, Santos G, De Lima F K and Da Silva Dias R F 2007 Flexible AC Transmission System, Power Electronics Handbook, Elsevier: 797-822 